\title{
Life is dynamic - dentistry a well
}

\author{
Jean-Francois Roulet \\ DDS, PhD, Prof hc \\ Professor \\ University of Florida, Gainesville, FL, USA

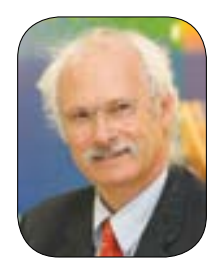

Dear Readers,

"Evidence based" is kind of a buzz word, when it comes to justify our doing as a dentist. This relates to, surgical techniques or most often to the material selection. The highest evidence comes from systematic reviews of random controlled clinical trials (RCCT). For these trials we like to see the longest possible observation times, having 10 or even better 15 years being considered the best. We all know, that the patient has a substantial influence on the outcome of clinical trials; therefore, all these studies deal with inclusion and most important exclusion criteria. The goal is to eliminate as much bias as possible. The thought behind this is that then the conditions under which the restoration or implant is subjected to clinical use are standardized. This is the point where my criticism starts. 1 am asking you: "Doyou live the same way as in the year 2000 or 2005?" Most probably not. Furthermore, we must ask ourselves was the science and art of dentistry the same 15 years ago?

This leads me to the main question which is in my mind: Are we teaching dentistry the right way? Trying to remember my student time, which was more than 40 years ago 1 think we were given a lot of facts. 1 perceived delving into anatomy, physiology or biochemistry very similar to learning a new language. Lots of terms (facts) to memorize and rules to learn. However, when it came to the clinic, we were taught clinical procedures. Slightly exaggerating, we were told how to do it, and if we had questions about the "why" they were not well received. My perception is that teaching these days is not much different. In the age of multiple choice tests, we train our students to look at facts and maybe traps in the questions, which is a good thing, because they are forced at least to read precisely. But the clinical education to my knowledge very often is still reduced to teaching procedures. Especially when grading is a necessity, the simplest way is to create standards and try to see how far away from the "ideal" standard the work to be graded is. Again, 1 try to remember the procedures we did when 1 was a student - and now 1 try to compare with what 1 am doing today, or in otherwords, 1 try to realize how much has changed in dentistry. My educated guess is that maybe $20 \%$ of what 1 havelearned as a student is still identical or valid. "Only as little?" you would say. 1 am correct, when it comes to the procedures, but some general principles have survived the aging process, and this leads me to the following thought. Once the student knows the basic facts, so he/she can think in our specialty, we better should teach two things: (1) principles and their application, and; (2) life long learning. Our live is changing so fast, even more with increasing speed, that if we are not able to constantly learn and adapt, we will be lost in the past. Looking at myself, l went from reading books, using paper and pencil and reference tables, to using the computer, learning different operating systems, numerous softwares, and searching the internet for information. 1 am experiencing the radical change in dental technology from the wax knife to the mouse and the CAD software, from investing, casting etc. to CAM. When 1 go to the physician, things are completely different. Before an array of data acquired with all kinds of technology are present, there is little talking with your doctor. The same in daily life: Cars do not have keys anymore, gas tanks no lid. We have forgotten how to read maps, because the navi hopefully tells us where to go and and and.... Knowing all this, should we really not give a lot of thinking to the way we are giving along knowledge, competence and wisdom? As editors we must ask the same question: 1s it sufficient to produce an excellent, high quality journal, or should we think about rendering a true service to our readers, controlled by the reader rather than the editor? The technologies would be available. Dear readers, please let me know your opinion. 